
Effectiveness on Treatment and Survival of Children Diagnosed with

\title{
Cancer in Iran
}

\author{
Azim Mehrvar (iD 1,2, ${ }^{\text {, }}$, Sara Aghdamizadeh ${ }^{1}$, Samaneh Soleimanizadeh ${ }^{1}$, Negin Jafariyan Laijani ${ }^{1,3}$, \\ Mohsen Rouzrokh ${ }^{1,4}$, Yasaman Sadeghi ${ }^{1,5}$, Narjes Mehrvar ${ }^{10}{ }^{1}$ and Mardawig Alebouyeh ${ }^{1}$ \\ ${ }^{1}$ Mahak Hematology Oncology Research Center, Mahak Hospital, Shahid Beheshti University of Medical Sciences, Tehran, Iran \\ ${ }^{2}$ AJA Cancer Epidemiology Research and Treatment Center, AJA University of Medical Sciences, Tehran, Iran \\ ${ }^{3}$ Department of Genetics, Islamic Azad University, Damghan Branch, Damghan, Iran \\ ${ }^{4}$ Pediatric Surgery Research Center, Research Institute for Children's Health, Shahid Beheshti University of Medical Sciences, Tehran, Iran \\ ${ }^{5}$ Department of Pathobiology, School of Public Health and Urology Research Center, Tehran University of Medical Sciences, Tehran, Iran \\ "Corresponding author: Mahak Hematology Oncology Research Center, Mahak Hospital, Shahid Beheshti University of Medical Sciences, Tehran, Iran. Email: \\ drazimmehrvar@yahoo.com
}

Received 2021 August 06; Revised 2022 January 12; Accepted 2022 January 31.

\begin{abstract}
Background: Childhood cancer is the second leading cause of death in children. Approximately, $40 \%$ of children with cancer need to be admitted to the intensive care unit (ICU), which plays a key role in their treatment procedure and overall survival.

Objectives: In this study, we aimed at evaluating the impact of ICU admission on pediatric patients' survival and prognosis at Mahak Pediatric Cancer Treatment and Research Center (MPCTRC), Tehran, Iran.

Methods: Amongst a total number of 2693 hospitalized patients who were referred to our center from March 2014 to September 2019, 674 patients younger than 14 years of age who were at least admitted once to the ICU were included in this study. All the collected data were analyzed using IBM-SPSS software version 22.

Results: Totally, $48.96 \%$ of patients were female and $51.03 \%$ of them were male. The most frequent types of cancer were central nervous system tumors (33.23\%) and Leukemia (18.99\%). Additionally, 43.62\% of admitted patients were in the 1- 4 age group, with the mean age of $1.5 \pm 0.30$. The mortality rate in ICU was $40.20 \%$ and the overall survival (OS) rate was $29.5 \% \pm 2.6$. Moreover, the OS in children with solid tumors and non-solid tumors were $27.1 \% \pm 5.5$ and $32.6 \% \pm 3.1$, respectively.

Conclusions: The results of this conducted study provide a complete report on the status of children admitted to the ICU ward in Mahak Hospital. Because of this fact that the most frequent type of cancer was CNS tumors and children with CNS tumors are usually admitted to the ICU in the late stages of their disease, the overall survival rate in our study was $29.80 \%$, which is lower than other studies.
\end{abstract}

\section{Background}

Pediatric cancer comprises $0.5 \%$ of all cancers worldwide and is the leading cause of death in children younger than 14 years old (1), with an incidence rate of 50 to 200 cases per one million population (2). However, recent improvements in treatment protocols have considerably increased the survival rate of children diagnosed with cancer (3).

Studies have indicated that approximately one out of every 285 children diagnosed with cancer is admitted to the pediatric intensive care unit (PICU) in the United States annually (4). Although the mortality rate of children admitted to the intensive care unit (ICU) because of childhood cancer is much higher than admitted non-cancerous children (5), over the past decades there have been significant advancements in the status of the former group (6). To be more precise, their overall survival rate increased to $80 \%$ and their mortality rate significantly decreased (7). Nevertheless, despite recent therapeutic advances in the treatment of children with cancer, the side effects of the treatment methods had led to the hospitalization of children $38 \%$ of them need to be admitted to the ICU for the first 3 years of their treatment $(7,8)$.

It is also reported that these children account for approximately $4.2 \%$ of all ICU hospitalizations and $11.4 \%$ of all ICU deaths (8).

The ICU ward plays a key role in improving the performance of treatment plans and increasing the life expectancy of hospitalized children. Therefore, it is consid- 
ered an integral part of the treatment protocol. Despite the importance of hospitalization children with cancer in the ICU (9) only a few studies have been conducted in this field around the world, especially in developing countries such as Iran and other neighboring countries (10).

Mahak Pediatric Cancer Treatment and Research Center (MPCTRC) is a multidisciplinary referral center that plays a crucial role in the research, diagnosis, management, and care of pediatric patients with cancer younger than 14 years old. Children with malignancy from Iran and other countries are offered free support and treatment by the Mahak Hospital, a non-governmental organization (NGO).

\section{Objectives}

In this study, we aimed at evaluating the influence of hospitalization in the ICU on the treatment and survival of pediatric cancer in the Mahak Hospital.

\section{Methods}

\subsection{Study Design}

This retrospective study was conducted on 674 children diagnosed with cancer who were admitted to the ICU of Mahak Hospital amongst a total number of 2693 hospitalized patients from March 2014 to September 2019.

\subsection{Data Collection}

All data was collected from patients' clinical documents including demographic information [name, gender, date of birth, and familial history of cancer] and clinical data [type of cancer, date of diagnosis, date of hospitalization, date of treatment, date of death (if applicable), status of patients at the time of ICU discharge, adverse drug reactions (ADR) (drug allergies, grade ADR and time of ADR), secondary cancer, relapse and metastasis, treatment protocols, tracheal intubation and, extubation, number of admissions to ICU, etc.

\subsection{Inclusion and Exclusion Criteria}

Because of the fact that according to WHO, childhood cancer is defined when a pediatric patient younger than 14 years old is diagnosed with cancer therefore, we included all the children who were under 15 years old at the time of diagnosis with any type of cancer who admitted to the ICU from March 2014 to September 2019 were included in this study. Patients with non-cancerous diseases such as anemia and thalassemia were excluded from this study.

\subsection{Survival and Prognosis}

To better understanding the effect of ICU admission on patients' lives, we evaluated overall survival(OS) rates with the Kaplan-Meier method. The Total-OS (TOS) was based on the time of diagnosis to the last follow-up of the patient and the ICU-OS calculated from the time of ICU admission to the last follow-up.

\subsection{Statistical Analysis}

All the collected data were entered in SPSS software version 22. Descriptive analysis was done using MannWhitney, Spearman correlation coefficient, Kaplan-Meier Log Rank. P-value less than 0.05 was considered as statistically significant.

\section{Results:}

\subsection{Patient's Demographic and Clinical Characteristics}

Totally, 674 children diagnosed with cancer were admitted to the ICU amongst a total number of 2693 hospitalized patients from March 2014 to September 2019. Among which $48.96 \%(n=330)$ were female and 51.03\% $(n=344)$ were male, (male to female ratio was 1/1.04). Patients' mean age was $2.54 \pm 0.93$ years, ranging from 5 days to 14 years. The majority of children were in the 1 to 4 years-old age group $(43.62 \%, n=293)$, and children younger than oneyear-old were the fewest ones $(11.12 \%, \mathrm{n}=75)$. The mean age of patients in their first ICU admission was $2.8 \pm 0.97$ years, ranging from 5 months to 19 years old. More information about patients' demographic characteristics are shown in Table 1.

The commonest malignancies amongst children were central nervous system tumors $(33.23 \%, \mathrm{n}=224$ ) and leukemia $(18.99 \%, \mathrm{n}=128)$. Additionally, Langerhans cell histiocytosis $(0.74 \%, \mathrm{n}=5)$ and germ cell tumors $(1.92 \%, \mathrm{n}$ $=13$ ) were the least frequent type of cancer among the children . Information about other types of cancer are shown in Table 1.

Duration of hospitalization to the ICU ranged from one day to 45 days, with a mean time of $7.24 \pm .044$ days.

\subsection{Patient's Status, Follow-up, and Survival}

At the time of patients' last follow-up(September 2019), 76 (11.30\%) children were off-treatment because of disease progression, 235 (34.90\%) were during their treatment procedure and unfortunately, 363 (53.90\%) patients died.

Regarding events in their treatment procedure from the time of disease diagnosis to the last follow-up, $20.62 \%$ $(n=139)$ and $5.34 \%(n=36)$ of patients experienced disease relapse and cancer metastasis, respectively, and 5 patients were diagnosed with secondary cancer. Of these events, 36 


\begin{tabular}{|c|c|c|c|}
\hline & Female & Male & Total \\
\hline \multicolumn{4}{|l|}{ Age group at the time of diagnosis } \\
\hline$<1$ & $43(13.03)$ & $32(9.30)$ & 75 (11.12) \\
\hline $1-4$ & $138(41.81)$ & $156(45.34)$ & $294(43.62)$ \\
\hline $5-9$ & $84(25.45)$ & $89(25.87)$ & $173(25.66)$ \\
\hline $10-14$ & $65(19.69)$ & $67(19.47)$ & $132(19.58)$ \\
\hline Mean age $(y)$ at the time of diagnosis & $2.52 \pm 0.91$ (years) & $2.56 \pm 0.94$ (years) & $2.54 \pm 0.93$ (years) \\
\hline \multicolumn{4}{|l|}{ Age group at the time of ICU admission } \\
\hline$<1$ & $17(5.15)$ & $13(3.77)$ & $30(4.45)$ \\
\hline $1-4$ & $138(41.81)$ & $134(38.95)$ & $272(40.5)$ \\
\hline $5-9$ & $90(27.27)$ & $99(28.77)$ & $189(28.04)$ \\
\hline $10-14$ & $72(21.81)$ & $82(23.83)$ & $154(22.84)$ \\
\hline$>15$ & $13(3.93)$ & $16(4.65)$ & $29(4.30)$ \\
\hline Mean age $(y)$ at the time of icu admission & $2.77 \pm 0.97$ (years) & $2.86 \pm 0.97$ (years) & $2.8 \pm 0.97$ (years) \\
\hline Type of diagnosis - solid tumors & $248(36.79)$ & $264(39.16)$ & $512(75.96)$ \\
\hline CNC tumors & $112(45.16)$ & $112(42.42)$ & $224(33.23)$ \\
\hline Neuroblastoma & $30(12.09)$ & $63(23.86)$ & $93(13.79)$ \\
\hline Sarcoma & $46(18.54)$ & $40(15.15)$ & $86(12.75)$ \\
\hline Renal tumors & $31(12.50)$ & $23(8.71)$ & $54(8.011)$ \\
\hline Retinoblastoma & $10(4.03)$ & $9(3.40)$ & $19(2.81)$ \\
\hline Hepatic tumors & $11(4.43)$ & $7(2.65)$ & $18(2.67)$ \\
\hline Germ cell tumor & $5(2.16)$ & $8(3.03)$ & $13(1.92)$ \\
\hline Langerhans cell histiocytosis & $3(1.20)$ & $2(0.75)$ & $5(0.74)$ \\
\hline Type of diagnosis - non-solid tumors & $75(11.2)$ & $87(12.90)$ & $162(24)$ \\
\hline Leukemia & $58(77.33)$ & $70(80.45)$ & $128(18.99)$ \\
\hline Lymphoma & $17(22.66)$ & $17(19.54)$ & $34(5.44)$ \\
\hline \multicolumn{4}{|l|}{ Status of patient at the time of ICU discharge } \\
\hline Alive & $203(30.12)$ & $200(29.67)$ & $403(59.79)$ \\
\hline Dead & $127(18.84)$ & $144(21.36)$ & $271(40.20)$ \\
\hline \multicolumn{4}{|l|}{ Status of patient at the time of last follow-up } \\
\hline Alive & $161(48.78)$ & $150(43.60)$ & $311(46.14)$ \\
\hline Dead & $169(51.21)$ & $194(56.39)$ & $363(54.74)$ \\
\hline \multicolumn{4}{|l|}{ Number of ICU admission } \\
\hline Once & $231(70.0)$ & $250(72.67)$ & $481(71.36)$ \\
\hline Twice & $56(16.96)$ & $71(20.63)$ & $127(18.84)$ \\
\hline More than three times & $43(3.03)$ & $23(6.68)$ & $66(9.79)$ \\
\hline
\end{tabular}

${ }^{a}$ Values are expressed as No. (\%). 
(5.34\%) cases of relapses, 8 (1.18\%) cases of metastasis, and one case of secondary cancer caused ICU admission in patients. More information about events based on each gender are shown in Table 2.

\begin{tabular}{lccc}
\hline $\begin{array}{l}\text { Table 2. Information About Disease Relapse, Metastasis, and Secondary Cancer } \\
\text { Events Based on Sex of Patient }(\mathrm{N}=674)^{\mathrm{a}}\end{array}$ & Female & Male & Total \\
\hline \multicolumn{4}{c}{} \\
\hline Events up to the last follow-up & $77(11.42)$ & $62(9.20)$ & $139(20.62)$ \\
Relapse & $19(2.82)$ & $17(2.52)$ & $36(5.34)$ \\
Metastasis & $3(0.45)$ & $2(0.30)$ & $5(0.74)$ \\
\hline Secondary cancer & & & \\
Events causing ICU admission & $18(2.67)$ & $18(2.67)$ & $36(5.34)$ \\
\hline Relapse & $5(0.47)$ & $3(0.45)$ & $8(1.18)$ \\
\hline Metastasis & $1(0.15)$ & 0 & $1(0.15)$ \\
\hline Secondary cancer & & & \\
\hline
\end{tabular}

a Values are expressed as No. (\%).

Amongst 363 dead patients, 271 (40.20\%) patients expired at the ICU ward. The 3-year and 5-year overall survival (from the time of their cancer diagnosis) were $40.4 \pm 2.4$ and $29.50 \pm 2.6$, respectively (Figure 1). Besides, their 3-year and 5-year overall survival (from the time of the first admission to the ICU) was $29.6 \pm 2.9$. More information about patients' overall survival are shown in table 3 and Figure 2.

Among 674 patients admitted to the ICU, 285 (44.0\%) patients were intubated, of whom 120 (17.83\%) and 66 (9.81\%) patients were diagnosed with CNS tumors and leukemia, respectively. Also, intubation was more frequent amongst male patients $(52.63 \%, \mathrm{n}=150)$ than female ones $(47.37 \%, \mathrm{n}=135)$.

In this study, 481 patients were admitted once and 127 patients were admitted twice to the ICU during their treatment procedure. Also, a total number of 66 patients were hospitalized at the ICU for more than 3 times. The maximum duration of hospitalization in the ICU was 45 days and the minimum duration of hospitalization was one day. The mean time of stay in ICU was (7.24 \pm .044$)$ days.

\section{Discussion}

The purpose of this study was to provide a complete report on the status of children admitted to the intensive care unit (ICU) at the Mahak Hospital. Due to the lack of research on the impact of ICU admission on children with cancer survival and outcome, especially in low- income countries such as Iran, this retrospective study could play an important role in evaluating these patients' quality of life.
Although patients were administrated to the ICU with different types of cancer, there was no significant relationship between the type of cancer and the number of ICU hospitalizations or duration of it.

In Iran, several studies have been conducted on palliative care of children with cancer who were admitted to the ICU. In addition, studies on the types of infections during the period of hospitalization in ICU have been conducted (11), but to the best of our knowledge, no similar study has been conducted on children with cancer admitted to the intensive care unit.

Leukemia seems to be the most common cancer amongst pediatric patients, accounting for approximately $25 \%$ of all childhood cancers (11) followed by CNS tumors which form $17 \%$ of it (11) which were also, the most frequent type of cancer amongst patients of the present study. However, the frequency of CNS tumors was higher than leukemia, because children with CNS tumors needed more admitted to the ICU than other cancers.

According to the world health organization (WHO), Leukemia and CNS tumors are also more frequent among children in the 1 to 4 -years-old age group (12), which is similar to our findings. Since Leukemia and CNS tumors are the most common type of cancers, the frequency of children in this age group is consequently higher than other age groups.

The mortality rate in the oncology ICU is higher than other ICU ranges (7).

The mortality rate in our study was $40.20 \%$ which is lower in the range of mortality from other studies $(7,13)$. The reason why the death rate in our study is lower than a study conducted in another low-income country (Pakistan) is that in our center, in contrast to that study, there are pediatric hematologist-oncologists who use the latest treatment protocols to cure children in the ICU (10). Nevertheless, the mortality rate in some reports from high- income countries is lower than the findings of this study due to the advances in the treatment procedures, ICU infection control, and other improvements in curing children diagnosed with cancer $(1,7,14,15)$

The aim of our study was to evaluate the survival rate of children with cancer who were admitted to the ICU. Unfortunately, the overall survival of children in our study was lower $(29.5 \% \pm 2.6)$ compared to those conducted in developed countries $(1,16)$.

There are various reasons for the reduced survival rate, of which the most important one could be postponed referral of children to the Mahak Hospital, so most of them were in the late stages of their disease and efforts to survive them could not be effective enough. Recent studies in high-income countries have shown overall survival rates of up to $80 \%$ in pediatric cancer patients admitted to the ICU 
MehrvarA et al.

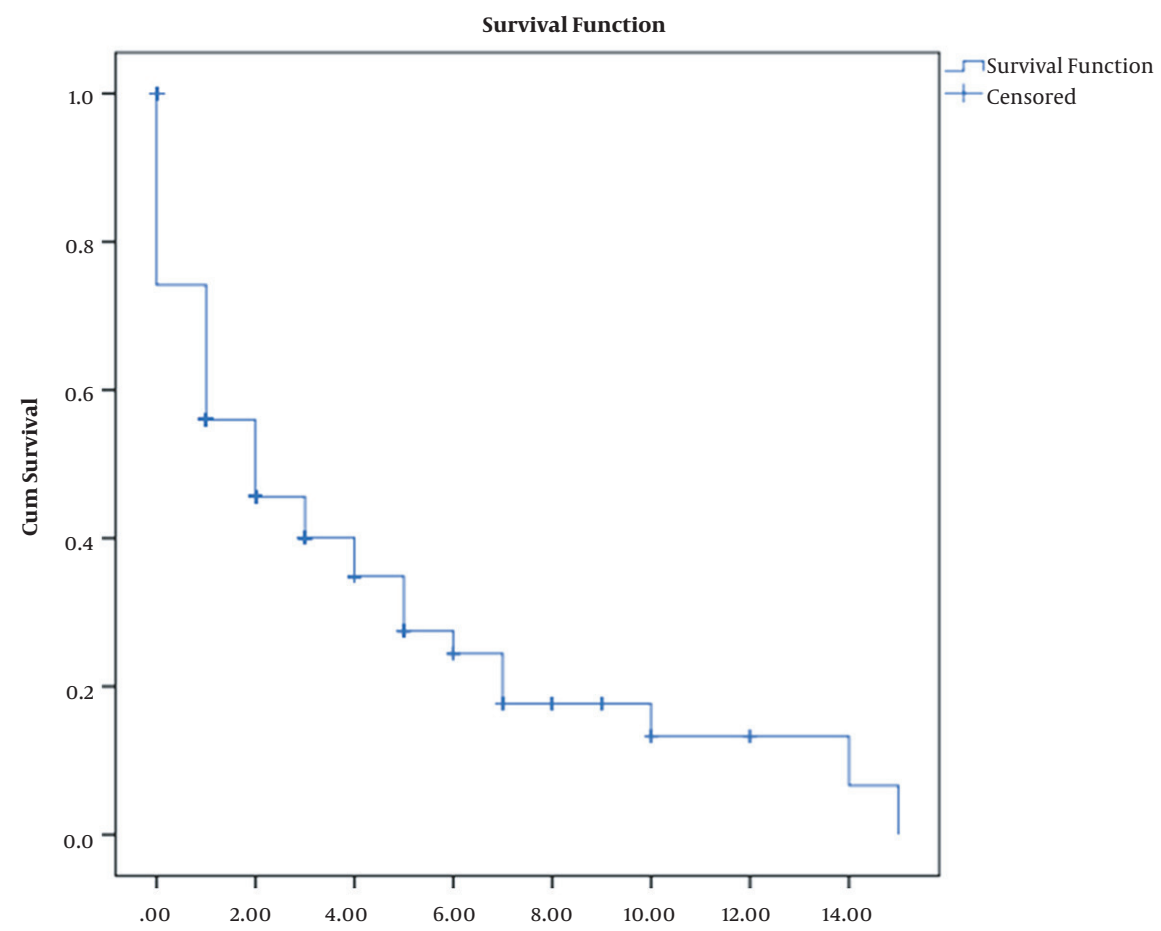

Figure 1. Total overall survival (TOS) in considered patients

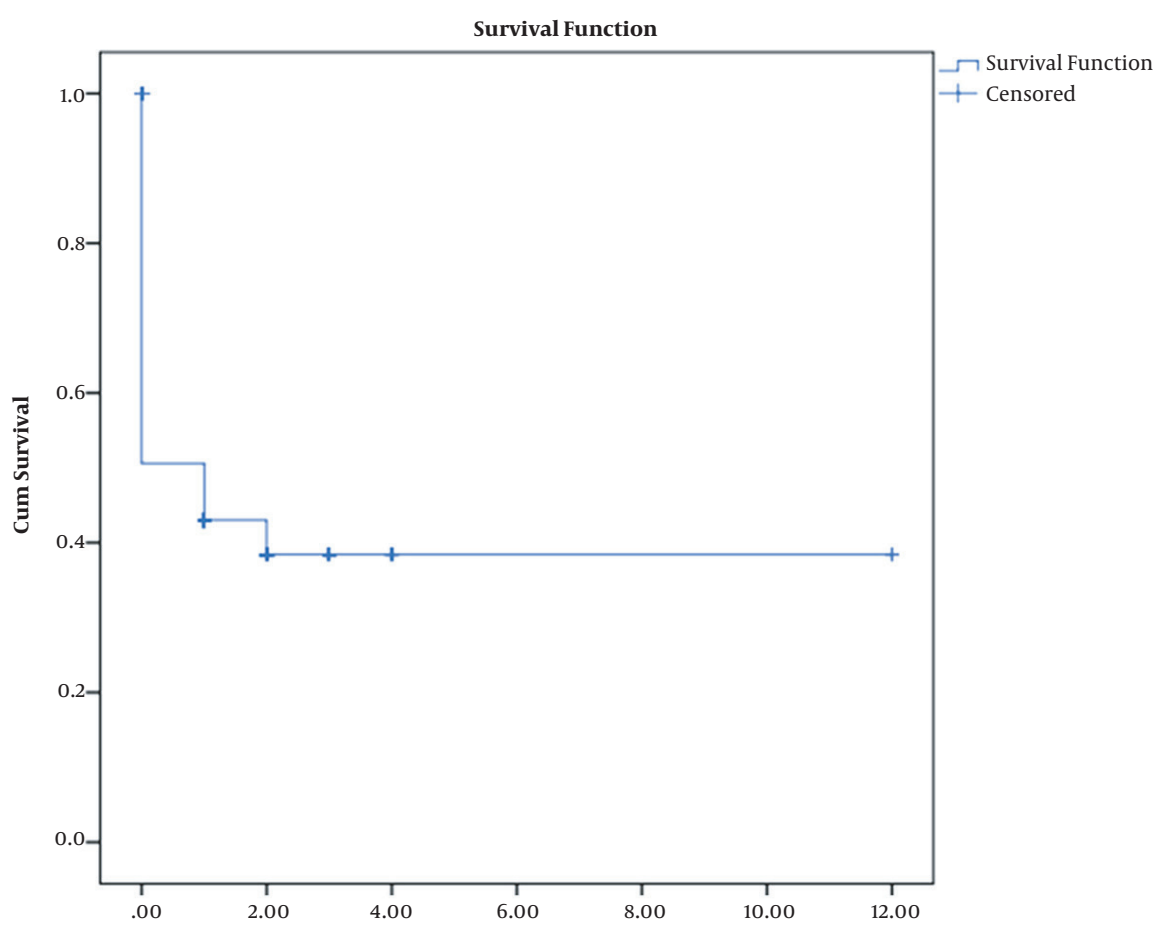

Figure 2. ICU-overall survival (from the time of ICU admission to the last follow-up) 


\begin{tabular}{|c|c|c|c|}
\hline \multirow{2}{*}{ Variables } & \multicolumn{3}{|c|}{ Overall Survival (OS) } \\
\hline & 1-year & 3-year & 5-year \\
\hline TOS (from the time of diagnosis to the last follow-up) & $70.9 \pm 1.8$ & $40.4 \pm 2.4$ & $29.5 \pm 2.6$ \\
\hline \multicolumn{4}{|l|}{ OS-1 based on type of cancer } \\
\hline Solid tumors & $78.0 \pm 3.4$ & $42.6 \pm 5.1$ & $27.1 \pm 5.5$ \\
\hline Non-solid tumors & $75.5 \pm 2.0$ & $44.0 \pm 2.7$ & $32.6 \pm 3.1$ \\
\hline \multicolumn{4}{|l|}{ OS-1 based on sex of patient } \\
\hline Female & $70.1 \pm 2.7$ & $39.2 \pm 3.5$ & $29.7 \pm 3.7$ \\
\hline Male & $71.6 \pm 2.5$ & $41.5 \pm 3.2$ & $29.5 \pm 3.6$ \\
\hline \multicolumn{4}{|l|}{ OS-1 based on the number of ICU admission } \\
\hline Once & $75.1 \pm 2.1$ & $44.4 \pm 2.9$ & $33.0 \pm 3.4$ \\
\hline Twice & 61.64 .4 & $38.3 \pm 4.9$ & $27.7 \pm 5.1$ \\
\hline More than three times & $64.3 \pm 6.1$ & $21.5 \pm 5.6$ & $13.9 \pm 5.1$ \\
\hline ICU-OS (from the time of ICU admission to the last follow-up) & $41.3 \pm 2.2$ & $29.6 \pm 2.9$ & $29.6 \pm 2.9$ \\
\hline \multicolumn{4}{|l|}{ OS-2 based on type of cancer } \\
\hline Solid tumors & $42.7 \pm 4.9$ & $28.3 \pm 6.9$ & $28.3 \pm 6.9$ \\
\hline Non-solid tumors & $43.9 \pm 2.6$ & $32.4 \pm 3.5$ & $32.4 \pm 3.5$ \\
\hline \multicolumn{4}{|l|}{ OS-2 based on sex of patient } \\
\hline Female & $40.3 \pm 3.0$ & $31.2 \pm 3.4$ & $31.2 \pm 3.4$ \\
\hline Male & $42.5 \pm 3.3$ & $28.7 \pm 4.4$ & $28.7 \pm 4.4$ \\
\hline \multicolumn{4}{|l|}{ OS-2 based on the number of ICU admission } \\
\hline Once & $48.6 \pm 2.6$ & $41.2 \pm 3.8$ & $41.2 \pm 3.8$ \\
\hline Twice & $32.7 \pm 4.5$ & $26.2 \pm 4.8$ & $26.2 \pm 4.8$ \\
\hline More than three times & $35.0 \pm 6.2$ & $6.6 \pm 4.2$ & $6.6 \pm 4.2$ \\
\hline
\end{tabular}

Abbreviations: TOS, total-overall survival; OS, overall survival.

${ }^{\text {a }}$ Values are expressed as percent.

(17). Also, the overall survival rate for solid tumors $(27.1 \%$ $\pm 5.5)$ was less than non-solid tumors $(32.6 \% \pm 3.1)$, which is similar to other studies and could be because of the fact that non-solid tumors have a better prognosis than solid ones $(1,18)$.

\subsection{Conclusions}

Despite the utilization of appropriate treatment protocols in order to cure children, the overall survival rate of children in this report was lower compared to other studies. The main reasons for that were the death of children during treatment and their late referral in the last stages of cancer. Considering that oncology ICU plays a more vital role in pediatric patients due to their disease condition, the overall survival in this type of ICU is usually lower than others, with higher rates of mortality. Finally, we suggest that a similar but multicenter study be conducted in order to assess survival and outcome of ICU admission amongst pediatric patients which could be more reliable.

\section{Acknowledgments}

This study has been supported by Mahak Pediatric Cancer Treatment and Research Center (MPCTR). We are thankful to our colleagues in Mahak Hospital especially from the ICU nurses who accompanied us well in collecting patients' information.

\section{Footnotes}

Authors' Contribution: Narjes Mehrvar: clinical studies; Negin Jafariyan Lahijani: concepts, design, definition of intellectual content, literature search, clinical studies, experimental studies, data acquistition an analysis, statistical analysis, manuscript preparation ;Yasaman 
Sadeghi: edditing, manuscript review; Narjes Mehrvar: design, data analysis, statistical analysis, manscript edditing and review; Mohsen Rouzrokh: concepts, clinical studies, manuscript review; Sara Aghdamizadeh and Samaneh Soleimanishakoor: data acquistition; Mardawig Alebouyeh: clinical studies; Azim Mehrvar: clinical studies, manuscript review, guarantor.

Conflict of Interests: The authors have no financial interest in this study.

Data Reproducibility: The data presented in this study are openly available in one of the repositories or will be available on request from the corresponding author by this journal representative at any time during submission or after publication. Otherwise, all consequences of possible withdrawal or future retraction will be with the corresponding author.

Funding/Support: Mahak Hematology Oncology Research Center (Mahak-HORC) supported this study.

\section{References}

1. Ali AM, Sayed HA, Mohammed MM. The Outcome of Critically Ill Pediatric Cancer Patients Admitted to the Pediatric Intensive Care Unit in a Tertiary University Oncology Center in a Developing Country: A 5-Year Experience. J Pediatr Hematol Oncol. 2016;38(5):355-9. doi: 10.1097/MPH.0000000000000523. [PubMed: 26907641].

2. Aleassa EM, Xing M, Keijzer R. Nanomedicine as an innovative therapeutic strategy for pediatric cancer. Pediatr Surg Int. 2015;31(7):611-6. doi: 10.1007/s00383-015-3683-2. [PubMed: 25690563].

3. Meyer S, Gottschling S, Biran T, Georg T, Ehlayil K, Graf N, et al. Assessing the risk of mortality in paediatric cancer patients admitted to the paediatric intensive care unit: a novel risk score? Eur J Pediatr. 2005;164(9):563-7. doi: 10.1007/s00431-005-1695-y. [PubMed: 15912385].

4. Cotoc C, An R, Klonoff-Cohen H. Pediatric Oncology and AnimalAssisted Interventions: A Systematic Review. Holist Nurs Pract. 2019;33(2):101-10. doi: 10.1097/HNP.0000000000000313. [PubMed: 30747779].

5. Heneghan JA, Pollack MM. Morbidity: Changing the Outcome Paradigm for Pediatric Critical Care. Pediatr Clin North Am. 2017;64(5):1147-65. doi: 10.1016/j.pcl.2017.06.011. [PubMed: 28941541]. [PubMed Central: PMC5654565].

6. Dalton HJ, Slonim AD, Pollack MM. MultiCenter outcome of pediatric oncology patients requiring intensive care. Pediatr Hematol Oncol. 2003;20(8):643-9. [PubMed: 14578035].
7. Wosten-van Asperen RM, van Gestel JPJ, van Grotel M, Tschiedel E, Dohna-Schwake C, Valla FV, et al. PICU mortality of children with cancer admitted to pediatric intensive care unit a systematic review and meta-analysis. Crit Rev Oncol Hematol. 2019;142:153-63. doi: 10.1016/j.critrevonc.2019.07.014. [PubMed: 31404827].

8. Zinter MS, DuBois SG, Spicer A, Matthay K, Sapru A. Pediatric cancer type predicts infection rate, need for critical care intervention, and mortality in the pediatric intensive care unit. Intensive Care Med. 2014;40(10):1536-44. doi: 10.1007/s00134-014-3389-2. [PubMed: 25023526]. [PubMed Central: PMC4177269].

9. Short SR, Thienprayoon R. Pediatric palliative care in the intensive care unit and questions of quality: a review of the determinants and mechanisms of high-quality palliative care in the pediatric intensive care unit (PICU). Transl Pediatr. 2018;7(4):326-43. doi: 10.21037/tp.2018.09.11. [PubMed: 30460185]. [PubMed Central: PMC6212394].

10. Khan Sial GZ, Khan SJ. Pediatric Cancer Outcomes in an Intensive Care Unit in Pakistan. J Glob Oncol. 2019;5:1-5. doi: 10.1200/JGO.18.00215. [PubMed: 30860953]. [PubMed Central: PMC6449076].

11. Hewitt M. Childhood Cancer Survivorship: Improving Care and Quality of Life. Washington DC, USA: National Academies Press; 2003. eng.

12. Howlader N, Noone AM, Krapcho M, Miller D, Bishop K, Altekruse SF. SEER Cancer Statistics Review, 1975 - 2013, Bethesda, MD. Natl Cancer Inst. 2016.

13. van Veen A, Karstens A, van der Hoek AC, Tibboel D, Hahlen K, van der Voort E. The prognosis of oncologic patients in the pediatric intensive care unit. Intensive Care Med. 1996;22(3):237-41. doi: 10.1007/BF01712243. [PubMed: 8727438].

14. Akhtar N, Fadoo Z, Panju S, Haque A. Outcome and prognostic factors seen in pediatric oncology patients admitted in PICU of a developing country. Indian J Pediatr. 2011;78(8):969-72. doi: 10.1007/s12098-0110391-3. [PubMed: 21437580].

15. Dursun O, Hazar V, Karasu GT, Uygun V, Tosun O, Yesilipek A. Prognostic factors in pediatric cancer patients admitted to the pediatric intensive care unit. J Pediatr Hematol Oncol. 2009;31(7):481-4. doi: 10.1097/MPH.ob013e3181a330ef. [PubMed: 19564740].

16. Hallahan AR, Shaw PJ, Rowell G, O'Connell A, Schell D, Gillis J. Improved outcomes of children with malignancy admitted to a pediatric intensive care unit. Crit Care Med. 2000;28(11):3718-21. doi: 10.1097/00003246-200011000-00030. [PubMed: 11098979].

17. Gutierrez YR, de Camargo B. Improved outcomes of children with malignancy admitted to a pediatric intensive care. Crit Care Med. 2001;29(6):1292. doi:10.1097/00003246-200106000-00050. [PubMed: 11395628].

18. Azoulay E, Mokart D, Pene F, Lambert J, Kouatchet A, Mayaux J, et al. Outcomes of critically ill patients with hematologic malignancies: prospective multicenter data from France and Belgium-a groupe de recherche respiratoire en reanimation onco-hematologique study. $J$ Clin Oncol. 2013;31(22):2810-8. doi: 10.1200/JCO.2012.47.2365. [PubMed: 23752112]. 